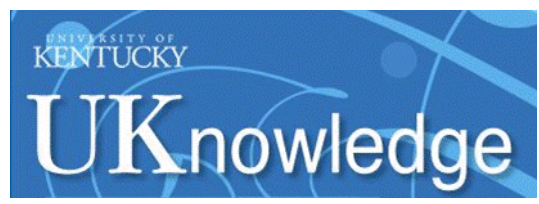

University of Kentucky

UKnowledge

10-1-1997

\title{
Do the Broad Emission Line Clouds See the Same Continuum That We See?
}

\author{
Kirk Korista \\ University of Kentucky \\ Gary J. Ferland \\ University of Kentucky, gary@uky.edu \\ Jack Baldwin \\ Cerro Tololo Inter-American Observatory, Chile
}

Follow this and additional works at: https://uknowledge.uky.edu/physastron_facpub

Part of the Astrophysics and Astronomy Commons, and the Physics Commons

Right click to open a feedback form in a new tab to let us know how this document benefits you.

\section{Repository Citation}

Korista, Kirk; Ferland, Gary J.; and Baldwin, Jack, "Do the Broad Emission Line Clouds See the Same Continuum That We See?" (1997). Physics and Astronomy Faculty Publications. 120.

https://uknowledge.uky.edu/physastron_facpub/120

This Article is brought to you for free and open access by the Physics and Astronomy at UKnowledge. It has been accepted for inclusion in Physics and Astronomy Faculty Publications by an authorized administrator of UKnowledge. For more information, please contact UKnowledge@lsv.uky.edu. 


\section{Do the Broad Emission Line Clouds See the Same Continuum That We See?}

Digital Object Identifier (DOI)

http://dx.doi.org/10.1086/304659

\section{Notes/Citation Information}

Published in The Astrophysical Journal, v. 487, no. 2, p. 555-559.

(C) 1997. The American Astronomical Society. All rights reserved. Printed in the U.S.A.

The copyright holder has granted permission for posting the article here. 
THE Astrophysical Journal, 487:555-559, 1997 October 1

(C) 1997. The American Astronomical Society. All rights reserved. Printed in U.S.A.

\title{
DO THE BROAD EMISSION LINE CLOUDS SEE THE SAME CONTINUUM THAT WE SEE?
}

\author{
KIRK Korista AND Gary Ferland \\ Department of Physics and Astronomy, University of Kentucky, Lexington, KY 40506 \\ AND \\ JACK BALDWIN \\ Cerro Tololo Inter-American Observatory, ${ }^{1}$ Casilla 603, La Serena, Chile \\ Received 1997 March 25; accepted 1997 May 9
}

\begin{abstract}
Recent observations of quasars, Mrk 335 and the HST quasar composite spectrum, have indicated that many quasars have remarkably soft ionizing continua $\left(f_{v} \propto v^{-2}, 13.6-100 \mathrm{eV}\right)$. We point out that the number of photons with $h v>54.4 \mathrm{eV}$ is insufficient to create the observed strengths of the $\mathrm{He}$ II emission lines. While the numbers of photons which energize $C$ IV $\lambda 1549$ and $O$ vi $\lambda 1034$ are sufficient, even the most efficiently emitting clouds for these two lines must each cover at least $20 \%-40 \%$ of the source. If the typical quasar ionizing continuum is indeed this soft, then we must conclude that the broad emission line clouds must see a very different (harder) continuum than we see. The other viable possibility is that the UV-EUV SED is double peaked, with the second peaking near $54 \mathrm{eV}$, its Wien tail being the observed soft X-ray excess.
\end{abstract}

Subject headings: line: formation - quasars: emission lines - ultraviolet: galaxies

\section{INTRODUCTION}

Since the recognition in 1963 of quasars as the most luminous entities in the universe, the continuum spectral energy distributions (SEDs) of quasars and active galactic nuclei have been studied extensively as a means of understanding these energetic objects (e.g., Malkan \& Sargent 1982; Sanders et al. 1989; Elvis et al. 1994). Of secondary importance is the fact that this continuum ionizes and excites the gas that emits the observed broad emission lines (Davidson 1977). Important to both is knowledge of the strength and shape of the ionizing continuum $(13.6 \mathrm{eV}-1 \mathrm{keV})$.

Determining the SED and number of ionizing photons has been difficult since most of the ionizing continuum lies in the unobservable portions of the spectrum. This continuum had to be inferred based upon the strengths of observed emission lines, such as He II $\lambda 1640$ (Mathews \& Ferland 1987), and others (Binette, Courvoisier, \& Robinson 1988; Krolik \& Kallman 1988; Binette et al. 1989; Clavel \& Santos-Lleó 1990; Zheng 1991; Gondhalekar 1992; Zheng, Fang, \& Binette 1992). However, with the advent of high-quality ground-based and space-based observations, the "unobservable gap" has begun to narrow substantially from the low- and high-energy ends of the ionizing spectrum. Based upon some of these recent contemporaneous broadband continuum observations, we will consider the continuum-broad emission line energy budgets of quasars and discuss whether or not these observed continuum SEDs might differ significantly from those incident upon the broad emission line clouds. The energy budget problem in its most general form was investigated by Netzer (1985).

Contemporaneous ground-based, IUE, HUT, and

\footnotetext{
${ }^{1}$ Operated by the Association of Universities for Research in Astronomy (AURA), Inc., under cooperative agreement with the National Science Foundation.
}

$B B X R T$ observations led Zheng et al. (1995) to propose that a comptonized accretion disk produces the continuum SED for Mrk 335. Their proposed continuum is one which, beginning near the rest-frame Lyman limit $(912 \AA)$, falls as $f_{v} \propto v^{-2}$ before meeting the X-ray power law (see also Mannheim, Schulte, \& Rachen 1995).

More recently, Zheng et al. (1997) presented a composite HST quasar spectrum. The quasar redshifts of $z \gtrsim 1$ allowed them to observe into the rest-frame far-UV. The analysis of Zheng et al. showed that an SED very similar to that inferred for Mrk 335 was consistent with their composite quasar spectrum, corrected for Galactic reddening and intergalactic absorption. Based upon the results of Zheng et al. and their quasar soft X-ray composite spectrum, Laor et al. (1997) also suggested the absence of an EUV bump.

This very soft UV bump may apply to high-redshift quasars as well. Multiwavelength observations by Bechtold et al. (1994), Elvis et al. (1994), and Kuhn et al. (1995) presented similar evidence for soft Lyman continua in these objects after correcting for Galactic reddening and intergalactic absorption. They inferred a natural connection between their data near the Lyman limit and the X-ray observations, the inferred slope being roughly that of the Comptonized accretion disk spectrum model of Zheng et al. (1995).

Such a soft ionizing continuum is surprising, especially in Mrk 335, which historically is known to be a relatively strong emitter of He II. Zheng et al. (1995) found $W_{\lambda}$ (He II $\lambda 1640) \approx 11.5 \AA$, while from the IUE archives Koratkar (1990) found an average equivalent with of about $13 \AA$ in this line. Over a 6 year period (1989-1990) Kassebaum et al. (1997) found $W_{\lambda}($ He II $\lambda 4686) \approx 22 \AA$ (see also Shuder 1981; Boroson \& Green 1992). In this paper, we will show that because of the paucity of $54.4 \mathrm{eV}$ photons, this SED is unlikely to account for the observed equivalent width of He II $\lambda 1640$ in Mrk 335 or in the composite HST quasar spectrum. Thus if the observed SEDs have been inferred 
correctly, the broad emission line clouds must see a different, harder continuum than we observe. We present the calculations in $\S 2$. A discussion of this conundrum and other possible explanations and a summary follow in $\S \S 3$ and 4.

\section{THE MAXIMUM He II $\lambda 1640$ EQUIVALENT WIDTH}

Our goal is to search for those cloud parameters (gas density and ionizing flux) that maximize the emission of He II $\lambda 1640$ and compare the energy emitted to that observed. Of course, one could produce an approximate theoretical limit to the He II equivalent width based upon the number of photons at energies greater than $54.4 \mathrm{eV}$, and assuming case B emissivity (MacAlpine 1981; MacAlpine et al. 1985; Mathews \& Ferland 1987). However, collisional excitation as well as optical depth effects will modify this result, and so we have chosen to run numerical simulations to identify the maximum theoretical limit to the He II $\lambda 1640$ equivalent width for a given incident SED. While we rely on the numerical results, we note that the predicted emission was essentially case B, except at high densities where the predicted flux was $\sim 2$ times larger and at high incident flux where thermalization at large optical depth depressed the emission below case $\mathrm{B}$.

Using the photoionization code CLOUDY (c90.03; Ferland 1996), we computed a grid of broad emission line spectra in the gas density-hydrogen ionizing flux plane (see Korista et al. 1997). Using a piecewise power law fitted to the spectrum of Mrk 335 inferred by Zheng et al. (1995), spectra from 841 clouds were computed. The $f_{v} \propto v^{-2}$ power law spanned from $1000 \AA$ to $100 \mathrm{eV} ; \alpha(100-700$ $\mathrm{eV}) \approx 1.6 ; \alpha(>700 \mathrm{eV}) \approx 1 ; \alpha_{\mathrm{ox}} \approx 1.46$. This value of $\alpha_{\mathrm{ox}}$ is rather soft for a Seyfert 1, but is typical for moderateluminosity QSOs such as those in the HST quasar composite (Wilkes et al. 1994; Laor et al. 1997). The gas density and ionizing flux spanned 7 orders of magnitude along each axis, and we assumed a column density of $10^{23} \mathrm{~cm}^{-2}$ and solar abundances. A larger column density will not increase the maximum equivalent width of $\mathrm{He}$ II $\lambda 1640$, and higher metal abundances will reduce its equivalent width due to the increased metal opacity (Ferland et al. 1996). In Figure 1

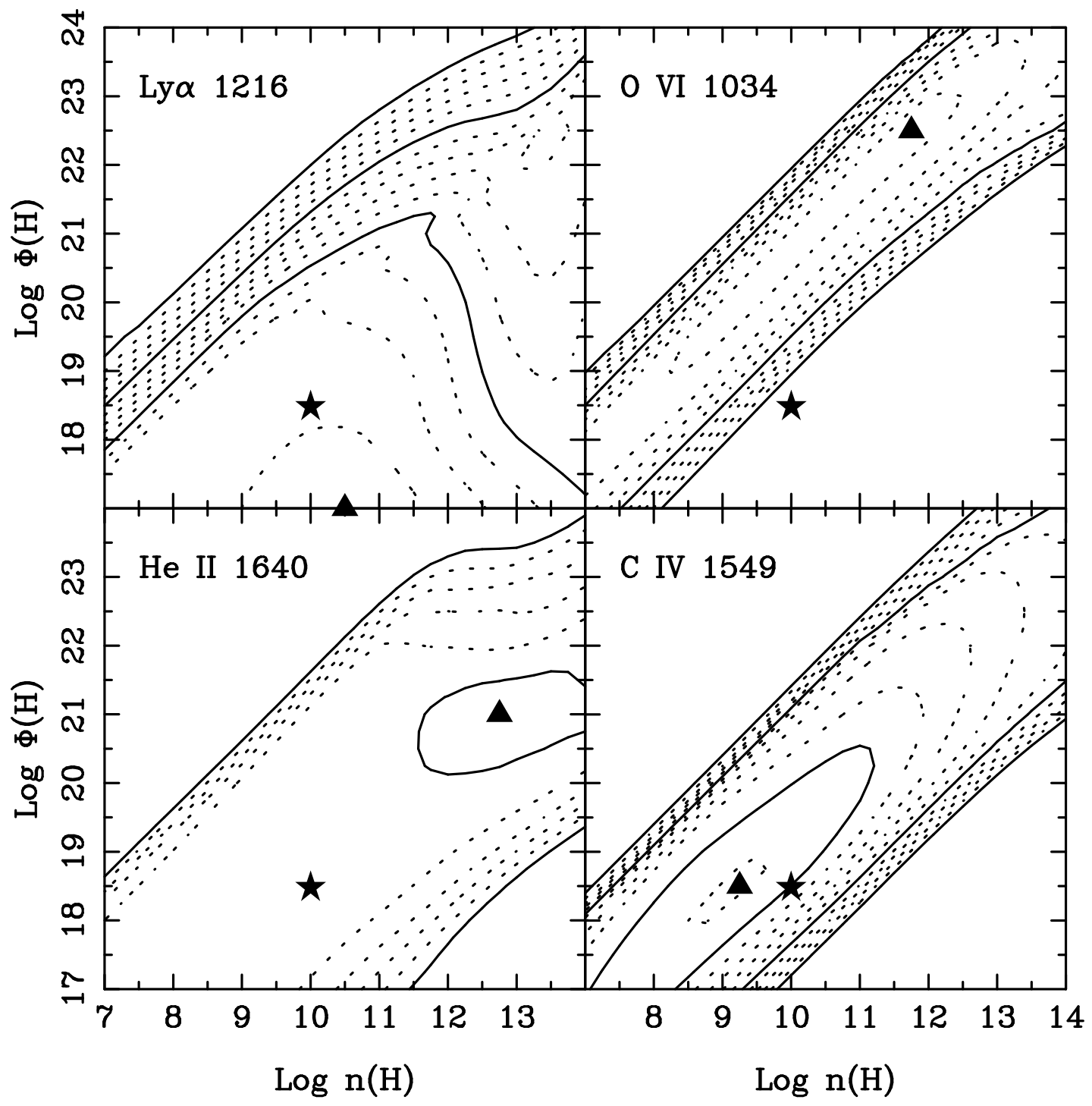

FIG. 1.-Contours of $\log W_{\lambda}$ for four emission lines for the soft SED grid are shown as a function of the hydrogen density and flux of hydrogen ionizing photons. Line strengths are expressed as logarithmic equivalent widths referenced to the incident continuum at $1215 \AA$ for full source coverage. The smallest decade contoured is $1 \AA$, each solid line is $1 \mathrm{dex}$, and dotted lines represent 0.2 dex steps. In each case the peak in the equivalent width distribution lies beneath the center of a solid triangle. The contours generally decrease monotonically from the peak to the $1 \AA$ contour. The solid star is a reference point marking the "standard BLR" parameters discussed by Davidson \& Netzer (1979). 
we plot in the gas density-hydrogen ionizing flux plane contours of four emission line equivalent widths, referenced to the incident continuum at $1215 \AA$, as in Baldwin et al. (1995) and Korista et al. (1997). The cloud covering fraction $\left(f_{c}\right)$ is assumed to be full in each case. A comparison of these line equivalent widths with those from the latter two references demonstrates the diminished emission-line equivalent widths resulting from the very soft spectrum employed here. Note that contour ratios in Figure 1 are equivalent to line flux ratios since the line equivalent widths are all referenced to the same continuum point at $1215 \AA$.

As indicated in Figure 1, these calculations show that this soft continuum generates a maximum equivalent width (with $f_{c}=1$ ) in He II $\lambda 1640$ of $\approx 13 \AA$, as measured at 1640 $\AA$. From Figure 1 , the cloud that emits He II $\lambda 1640$ optimally has $\log n_{\mathrm{H}} \approx 12.75$ and $\log \Phi(H) \approx 21$, corresponding to $\log U(H) \approx-2.25$. Here $\Phi(H)$ is the hydrogen ionizing photon flux and $U(H) \equiv \Phi(H) /\left(n_{\mathrm{H}} c\right)$. The observed equivalent widths in this line in Mrk 335 and the composite $H S T$ quasar spectrum are $\approx 11.5 \AA$ and $\approx 4.5 \AA$, respectively. The Mrk 335 measurement contains a minority narrow line contribution, but this matters little since sufficient numbers of $54.4 \mathrm{eV}$ photons still need to emerge from the nucleus to ionize the narrow line clouds. The case of Mrk 335 would require nearly full global coverage of the source by these optimally emitting $\mathrm{He}$ II clouds. However, if the column densities of these particular clouds were comparable to those expected for typical broad line clouds $\left(10^{22-24} \mathrm{~cm}^{-2}\right)$, this shell would be opaque between $13.6 \mathrm{eV}$ and a few $\mathrm{keV}$, while such absorption is never seen. In addition, the presence of strong $O$ VI $\lambda 1034$ and significant intercombination line strengths in the spectrum of Mrk 335 and the quasar composite spectrum require populations of clouds with other parameters. Thus, the emitting clouds of the BLR cannot all be those that emit He II optimally (see Fig. 1), and an ensemble of BLR clouds exposed to this soft continuum is more likely (e.g., Baldwin et al. 1995). Integrating over an ensemble of clouds leads to predicted $\mathrm{He}$ II $\lambda 1640$ equivalent widths of $\approx 3-4 \AA$ for $f_{c}=0.5$, much lower than the observed equivalent width in Mrk 335. Thus, the inferred SED at $54.4 \mathrm{eV}$ of Mrk 335 is too weak by factors of several.

For the HST quasar composite we can consider statistical arguments concerning the cloud covering fraction. Smith et al. (1981), Kinney et al. (1985), Antonucci, Kinney, \& Ford (1989), Koratkar, Kinney, \& Bohlin (1992), and others searched for and did not find intrinsic Lyman limit absorption in the spectra of quasars, arguing for cloud covering fractions of $\sim 10 \%$. For this covering fraction and the predicted $W_{\lambda}($ He II $\lambda 1640) \approx(6-8 \AA)\left(f_{c}\right)=0.6-0.8 \AA$ the HST quasar composite spectrum's SED at $54.4 \mathrm{eV}$ is at least 5 times too weak; covering fractions of $56 \%-75 \%$ are required.

These calculations assumed thermal intrinsic line widths. If significant turbulence or streaming is present within the emitting gas, then the line optical depths will diminish (perhaps along preferred directions). However, we find that the introduction of nonthermal line widths does not change the maximum equivalent width of $\mathrm{He}$ II $\lambda 1640$, but merely shifts this maximum to clouds exposed to higher ionizing fluxes.

We conclude that this soft SED cannot account for the observed strengths of the He II emission lines in quasars and AGNs, at least under the usual assumption that the cloud covering fraction is less than 1 .

\section{DISCUSSION}

\subsection{Incident EUV Flux Brighter Than Observed?}

Either the inferred continua are incorrect or else the clouds see a brighter EUV bump than we do. The latter is possible if the clouds are located in a flattened geometry, for example, and we view the quasar preferentially from above in the presence of an intrinsically anisotropic continuum. The predicted SEDs of simple accretion disk models do predict the disk to become harder at larger inclinations due to relativistic effects (Sun \& Malkan 1989). Scenarios such as this have been discussed before for broad line clouds, on theoretical bases (e.g., Netzer 1987), as well as observational ones (Wanders et al. 1995; Ferland et al. 1996; Goad \& Wanders 1996). An ionizing photon deficit became apparent more than a decade ago in the observations of extended narrow line regions of Seyfert galaxies (Neugebauer et al. 1980; Wilson, Ward, \& Haniff 1988; Penston et al. 1990); a comprehensive review of this problem is given by Binette, Fosbury, \& Parker (1993). However, this is the first time that observations have provided such a straightforward and stringent test for the broad emission line clouds. If this is true, it has important consequences for our understanding of quasars.

\subsection{Is the UV-EUV Bump "Double Peaked"?}

It is to be emphasized that the spectrum of an AGN or quasar has never been observed at $54 \mathrm{eV}$ in the rest frame. Thus, a second possibility is that the SED at energies of $\sim 40-300 \mathrm{eV}$ is rather fortuitously dominated by a second, powerful, EUV bump, peaking near $54 \mathrm{eV}$. In order to power the He II line in Mrk 335, it would have to contain energy comparable to the "classical" UV bump. There is evidence for such power in the EUV in at least one object. Mrk 478 is $1-3$ times brighter in $v L_{v}$ at $\sim 120 \mathrm{eV}$ than it is at $1300 \AA$ (Gondhalekar et al. 1994; Marshall et al. 1996). At higher energies, viz., $\sim 200-400 \mathrm{eV}$, a soft X-ray excess has been observed in many AGNs and quasars (e.g., Saxton et al. 1993). Perhaps this excess is not the tail of the classical UV bump (or associated accretion disk), but the Wien tail of a separate EUV bump. This EUV bump would have to lie just beyond the soft Lyman continuum which extends to at least $30 \mathrm{eV}$ in the composite $H S T$ quasar spectrum, and its Wien tail would be the observed soft X-ray excess. A single blackbody with $T_{\mathrm{BB}} \sim 2 \times 10^{5} \mathrm{~K}$ might accomplish this. The origin of such a component is unclear but could be the result of reprocessing (Czerny \& Życki 1994), or a large He II Lyman discontinuity in emission arising from the accretion disk (Hubeny \& Hubeny 1997).

\subsection{Another Energy Source?}

Another possibility is that another source of energy is available to the clouds other than photoionization from the central source, such as has been suggested for some of the the Fe II emission in quasars (Collin-Souffrin 1986; Joly 1987). This does not seem likely for He II , however, based upon the results of the line-continuum reverberation campaigns for AGNs (e.g., Korista et al. 1995), including Mrk 335 (Kassebaum et al. 1997), that show a strong correlation between the strength of He II and the continuum.

\subsection{Low Column Density He II-emitting Clouds?}

In $\S 2$ we concluded that even if there exists a special type of He II-emitting cloud population that fully covers the 
continuum source, it would be opaque to ionizing photons for standard broad emission line cloud column densities $\left[N(H) \approx 10^{22-24} \mathrm{~cm}^{-2}\right]$. Our calculations show that the clouds which emit He II $\lambda 1640$ optimally (Fig. 1) barely reproduce the observed equivalent width in Mrk 335 with a minimum column density $N(H) \approx 10^{20.3}$ and full source coverage. Such clouds are optically thick between $54.4 \mathrm{eV}$ and $\sim 200 \mathrm{eV}$ but are optically thin at all other energies. With the exception of those lines that are emitted in the $\mathrm{He}^{++}$zone, such a $\mathrm{He}^{+}$Lyman continuum filter would have relatively minor effects on the emitted spectrum of clouds that lie outside the filter. C IV $\lambda 1549$ would emit at roughly two-thirds strength in clouds that lie outside the filter. Thus in this way, well-layered clouds with chosen parameters would allow the integrated covering fraction of the BLR to be greater than 1, and the observed $\mathrm{He}$ II strength could be reconciled with the soft ionizing SED.

There are at least two problems with this scenario, however. First, it is improbable that this soft ionizing SED can simultaneously account for the observed He II $\lambda 1640$ and $\mathrm{O}$ VI $\lambda 1034$ equivalent widths. The observed $\mathrm{O}$ VI (broad line) equivalent widths of $23 \AA$ in Mrk 335 and $16 \AA$ in the quasar composite require the clouds that emit $\mathrm{O}$ VI most efficiently (Fig. 1) to cover $30 \%$ of the source in Mrk 335 and typically $20 \%$ of the source in the quasar composite spectrum. These same clouds must have larger ionization parameters and column densities $\left(\sim 10^{22-23} \mathrm{~cm}^{-2}\right)$ than the optimal He II-emitting clouds, and thus be completely opaque to the $\mathrm{He}^{+}$Lyman continuum out to a few $\mathrm{keV}$. Since no $\mathrm{O}$ VI is emitted in the optimal He II-emitting clouds (Fig. 1), the $\mathrm{O}$ VI emitters must lie closer to the continuum source than the He II-emitting shell (some of the $\mathrm{O}$ VI emission could originate outside the $\mathrm{He}$ II-emitting shell, but at $\lesssim \frac{1}{3}$ efficiency due to the shell's opacity). In the case of Mrk 335, 30\% of the $\mathrm{He}^{+}$Lyman continuum photons that were required to illuminate the He II-emitting shell would be absorbed by the $\mathrm{O}$ vI-emitting clouds inside the shell. The equivalent width of $\mathrm{He}$ II $\lambda 1640$ emitted in the $\mathrm{O}$ VI clouds amounts to $0.3 \times 8 \AA$, not quite enough to account for the deficit of $\mathrm{He}$ II emission in the shell due to the opacity of the $\mathrm{O}$ vi clouds.

A related problem is the observed $\mathrm{C}$ IV equivalent widths. As a bolometer C IV 21549 is less sensitive to the gas abundances. Its observed equivalent widths of $50 \AA$ and $69 \AA$ in Mrk 335 and the quasar composite spectrum require the most optimally emitting C IV clouds (Fig. 1) to cover $28 \%$ and $38 \%$ of the source, respectively. The required source coverages become larger in the presence of less efficient $\mathrm{C}$ IV-emitting clouds and larger still in the presence of an interior He II-emitting shell. However, in contrast to He II $\lambda 1640$, significant nonthermal gas motions would increase the peak equivalent widths of $\mathrm{C}$ IV and $\mathrm{O}$ VI, reducing somewhat their required cloud covering fractions.
A final problem with this scenario is that if the bulk of $\mathrm{He}$ II is emitted in this special set of low column density clouds, their response to continuum variations would likely be small or even negative, and certainly not strongly positive, contrary to what has been observed in Mrk 335 (Kassebaum et al. 1997) and other Seyfert 1 galaxies (Clavel et al. 1991).

\subsection{Possible Pitfalls in the Interpretations of the Observations}

It is possible that the far-UV continuum of the HST quasar composite was not correctly inferred. Significant corrections for intervening absorption had to be made to the data. Zheng et al. (1997) claim an uncertainty in their $350-1050 \AA$ slope of \pm 0.15 ; however, even if this were an underestimate, they find consistency with previous measurements of far-UV slopes in other quasars.

It should also be noted that the composite $H S T$ quasar spectrum is composed of a varying number of constituents at different wavelengths. Specifically, while most objects in the composite contributed to $\mathrm{He}$ II $\lambda 1640$, a diminishing number contributed to wavelengths shorter than about $1000 \AA$. Thus, determining how representative the composite spectrum is of the individual quasars is not straightforward. In particular, it is unclear from a composite spectrum how the He II strengths in individual objects correlate with the far-UV SEDs. Although in the case of Mrk 335 there was minimal far-UV spectral coverage, the contemporaneous multiwavelength nature of these observations mitigates the "composite" problem, and it demonstrates a larger deficit of $54.4 \mathrm{eV}$ photons.

\section{SUMMARY}

In summary, recent observations of quasars have indicated many quasars have remarkably soft ionizing continua, so that the number of $54.4 \mathrm{eV}$ photons is insufficient to create the observed strengths of the $\mathrm{He}$ II emission lines. While the numbers of photons which energize C IV $\lambda 1549$ and $\mathrm{O}$ VI $\lambda 1034$ are sufficient, even the most efficiently emitting clouds for these two lines must each cover at least $20 \%-40 \%$ of the source. If the soft ionizing continua conclusion holds up under further scrutiny and the phenomenon is common, then we consider the most likely conclusion to be that the broad emission line clouds must see a very different (harder) continuum than we see. The other viable option would seem to be that the UV-EUV SED is double peaked, with the second peaking near $54 \mathrm{eV}$, its Wien tail being the observed soft X-ray excess.

This work was supported by the NSF (AST 93-19034), NASA (NAGW-3315, NAG-3223), and STScI grant GO2306. We thank an anonymous referee for his or her constructive comments.
Antonucci, R. R. J., Kinney, A. L., \& Ford, H. C. 1989, ApJ, 342, 64

Baldwin, J. A., Ferland, G. J., Korista, K. T., \& Verner, D. 1995, ApJ, 455, L119

Bechtold, J., Elvis, M., Fiore, F., Kuhn, O., Cutri, R. M., McDowell, J. C., Rieke, M., Siemiginowska, A., \& Wilkes, B. 1994, AJ, 108, 374

Binette, L., Courvoisier, T. J.-L., \& Robinson, A. 1988, A\&A, 190, 29

Binette, L., Fosbury, R. A., \& Parker, D. 1993, PASP, 105, 1150

Binette, L., Prieto, A., Szuszkiewicz, E., \& Zheng, W. 1989, ApJ, 343, 135

Boroson, T. A., \& Green, R. F. 1992, ApJS, 80, 109

Clavel, J., et al. 1991, ApJ, 366, 64

Clavel, J., \& Santos-Lleó, M. 1990, A\&A, 230, 3

Collin-Souffrin, S. 1986, A\&A, 166, 13

\section{REFERENCES}

Czerny, B., \& Życki, P. T. 1994, ApJ, 431, L5

Davidson, K. 1977, ApJ, 218, 20

Davidson, K., \& Netzer, H. 1979, Rep. Prog. Phys., 51, 715

Elvis, M., et al. 1994, ApJS, 95, 1

Ferland, G. J. 1996, Hazy, a Brief Introduction to Cloudy, Univ. Kentucky Phys. Dept. Internal Rept

Ferland, G. J., Baldwin, J. A., Korista, K. T., Hamann, F., Carswell, R. F., Phillips, M. M., Wilkes, B. J., \& Williams, R. E. 1996, ApJ, 461, 683

Goad, M. R., \& Wanders, I. 1996, ApJ, 469, 113

Gondhalekar, P. M. 1992, MNRAS, 255, 663

Gondhalekar, P. M., Kellet, B. J., Pounds, K. A., Mathews, L., \& Quenby, J. J. 1994, MNRAS, 268, 973 
Hubeny, I., \& Hubeny, V. 1997, ApJ, 484, L37

Joly, M. 1987, A\&A, 184, 33

Kassebaum, T. M., Peterson, B. M., Wanders, I., Pogge, R. W., Bertram, R., \& Wagner, R. M. 1997, ApJ, 475, 106

Kinney, A. L., Huggins, P. J., Bregman, J. N., \& Glassgold, A. E. 1985, ApJ, 291,128

Koratkar, A. 1990, Ph.D. thesis, Univ. Michigan

Koratkar, A. P., Kinney, A. L., \& Bohlin, R. C. 1992, ApJ, 400, 435

Korista, K. T., Baldwin, J. A., Ferland, G. J., \& Verner, D. A. 1997, ApJS, 108,401

Korista, K. T., et al. 1995, ApJS, 97, 285

Krolik, J. H., \& Kallman, T. R. 1988, ApJ, 324, 714 Kuhn, O., Bechtold, J., Cutri, R., Elvis, M., \& Riecke, M. 1995, ApJ, 438,

Laor, A., Fiore, F., Elvis, M., Wilkes, B. J., \& McDowell, J. C. 1997, ApJ, 477, 93

MacAlpine, G. M. 1981, ApJ, 251, 465

MacAlpine, G. M., Davidson, K., Gull, T. R., \& Wu, C.-C. 1985, ApJ, 294, 147

Malkan, M. A., \& Sargent, W. L. W. 1982, ApJ, 254, 22

Mannheim, K., Schulte, M., \& Rachen, J. 1995, A\&A, 303, L41

Marshall, H. L., Carone, T. E., Shull, J. M., Malkan, M. A., \& Elvis, M. 1996, ApJ, 457, 169
Mathews, W. G., \& Ferland, G. J. 1987, ApJ, 323, 456

Netzer, H. 1985, ApJ, 289, 451

Netzer, H. 1987, MNRAS, 225, 55

Neugebauer, G., et al. 1980, ApJ, 238, 502

Penston, M. V., et al. 1990, A\&A, 236, 53

Sanders, D. B., et al. 1989, ApJ, 347, 29

Saxton, R. D., Turner, M. J. L., Williams, O. R., Stewart, B. C., Ohashi, T., \& Kii, T. 1993, MNRAS, 262, 63

Shuder, J. M. 1981, AJ, 86, 1595

Smith, M. G., Carswell, R. F., Whelan, J. A. J., Wilkes, B. J., Boksenberg, A., Clowes, R. G., Savage, A., Cannon, R. D., \& Wall, J. V. 1981, MNRAS, 195, 437

Sun, W.-H., \& Malkan, M. A. 1989, ApJ, 346, 68

Wanders, I., et al. 1995, ApJ, 453, L87

Wilkes, B. J., Tananbaum, H., Worrall, D. M., Avni, Y., Oey, M. S., \& Flanagan, J. 1994, ApJS, 92, 53

Wills, B., Netzer, H., \& Wills, D. 1985, ApJ, 288, 94

Wilson, A. S., Ward, M. J., \& Haniff, C. A. 1988, ApJ, 334, 121

Zheng, W. 1991, ApJ, 382, L55

Zheng, W., et al. 1995, ApJ, 444, 632

Zheng, W., Fang, L.-Z., \& Binette, L. 1992, ApJ, 392, 74

Zheng, W., Kriss, G. A., Telfer, T. C., Grimes, J. P., \& Davdison, A. F. 1997, ApJ, 475, 469 